1999 Particle Accelerator Conf., NYC, NY, 3/29-4/2/99.

\title{
BARRIER CAVITIES IN THE BROOKHAVEN AGS*
}

\author{
$\underline{\text { M. Blaskiewicz }}^{\dagger}$,J.M. Brennan. T. Roser, K. Smith, \\ R. Spitz, A. Zaltsman, BNL, Upton, NY 11973, USA \\ M. Fujieda, Y. Iwashita, A. Noda, Kyoto University, Gokanosho, Uji,Kyoto 61 1, JP \\ M. Yoshii, KEK, 1-1 Oho, Tsukuba 305, JP \\ Y. Mori, C. Ohmori, Y. Sato, KEK - Tanashi, Tanashi 188, JP
}

\section{Abstract}

In collaboration with KEK two barrier cavities, each generating $40 \mathrm{kV}$ per turn have been installed in the Brookhaven AGS. Machine studies are described and their implications for high intensity operations are discussed.

\section{INTRODUCTION}

During the 1998 high energy physics run the rf system in the AGS Booster ran with harmonic number $h=1$, and the AGS ran with $h=6$. This allowed for six Booster transfers per AGS cycle, even though the machine circumferences are in the ratio 1:4. Running the Booster with $h=1$ opened up the possibility of emittance conserving barrier bucket manipulations using two dedicated barrier cavities. The controls were set up to allow barrier cavity operation in a "pulse stealing" mode wherein the machine settings for the production cycle were changed to those appropriate for barrier operations for a few machine cycles and then switched back to the production cycle settings. This allowed for machine studies and hardware development over the entire run.

The principles of barrier cavity operation have been described elsewhere[ $1,2,3]$. This paper focuses on hardware configurations and conclusions.

\section{EXPERIMENTAL SETUP}

The cavity and amplifier parameters are summarized in Table 1 . As is clear from the table the two devices are quite different. The large $R / Q$ of the cavity supplied by KEK allows the use of a small amplifier but requires beam loading compensation at moderate beam currents. The feedforward system is broad band with a full turn of delay [4]. The voltage waveforms without beam, corrected for uncompensated capacitive dividers, are shown in Figure 1. Dynamical implications are considered next.

Let $T_{0}$ and $E_{0}$ be the revolution period, and synchronous energy, respectively. Denote the arrival time of a proton as $n T_{0}+\tau$ on the $n$th turn and lict $\delta=E-E_{U}$ be its relative energy deviation. The proton equations of motion are derivable from the Hamiltonian

$$
H(\tau, \delta)=\frac{\eta \delta^{2}}{23^{2} E_{0}}-q f_{\Gamma} \int_{0}^{\tau} V\left(t^{\prime}\right) d t^{\prime} .
$$

\footnotetext{
- Work supported by US DOE

†Email: mmb@bni.gov
}

Table 1: Cavity parameters

\begin{tabular}{|c|c|c|}
\hline Parameter & BNL cavity & KEK cavity \\
\hline gaps per cavity & 4 & 4 \\
$f_{\text {res }}$ & $2.6 \mathrm{MHz}$ & $1.0 \mathrm{MHz}$ \\
$R / Q$ per gap & $250 \Omega$ & $2500 \Omega$ \\
$Q$ & 40 & 0.6 \\
coupling & single ended & push-pull \\
amplifier rating & $600 \mathrm{~kW}$ & $30 \mathrm{~kW}$ each \\
beam loading correction & none & feed-forward \\
\hline
\end{tabular}

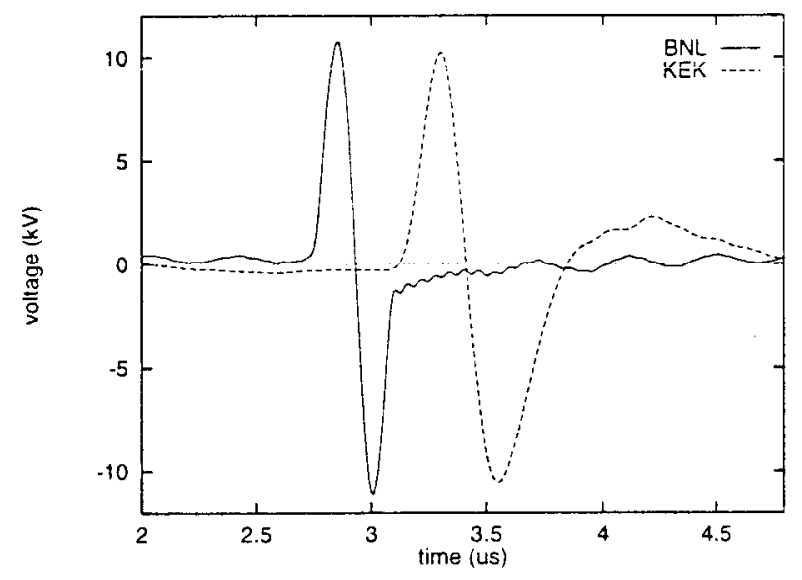

Figure 1: Gap voltage without beam.

where $\eta$ is the frequency slip factor, $\beta=v / c, q$ is the proton charge, and $f_{0}$ is the revolution frequency. The equations of motion are $d \tau / d t=\partial H / \partial \dot{\delta}$ and $d \delta / d t=$ $-\partial H / \partial \tau$. The integral of the voltage waveform is proportional to the longitudinal potential well and the familiar pictures of introductory mechanics are applicable.

The voltage integrals without beam are shown in Figure 2. Protons are repelled from high potential regions and undergo stable oscillations in low potential regions. Measurements were made using low intensity small emittance injected bunches to map out the potential wells. It was found that potential in the off-pulse region of the BNL cavity is flatter than show in the figure, which is consistent with the measured gap current for this cavity. The local minima in the $\mathrm{KEK}$ integral appear to be real.

Figure 3 shows the voltage waveforms of the BNL and KEK cavities along with the beam current. The oscillation 


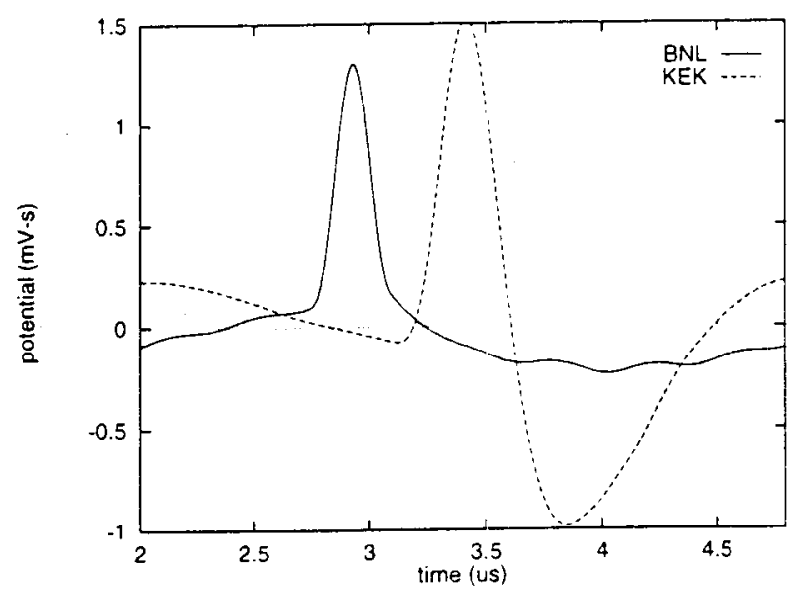

Figure 2: Integral of gap voltage without beam.

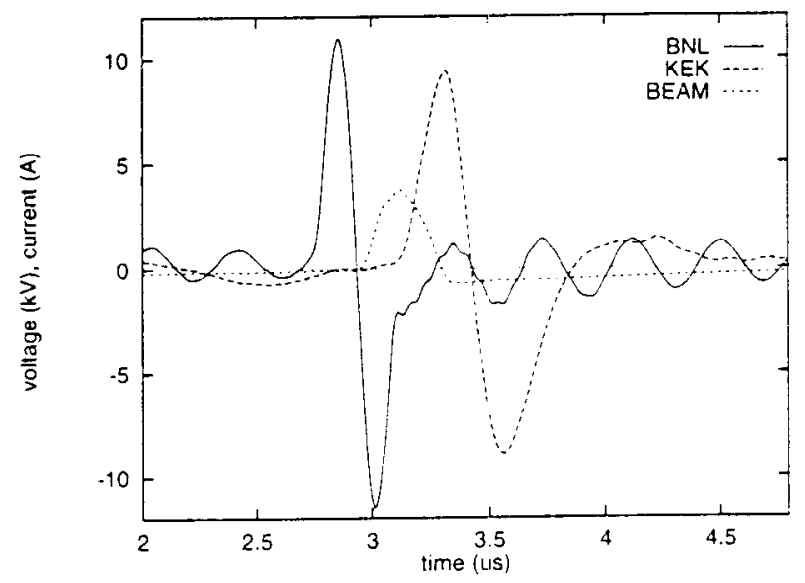

Figure 3: Gap voltage with beam and beam current. The feedforward on the KEK cavity is optimized.

in the BNL waveform is at the cavity resonant frequency with a decay rate consistent with the measured $Q$. The bunch contained $8.5 \times 10^{12}$ protons; about the number required for the barrier cavity if system to compete with the traditional rf system. Figure 4 shows the voltage integrals with beam.

\section{MULTIPLE TRANSFERS}

Controls for the barrier bucket system allowed multiple transfers, time dependent adjustment of the relative phase of the barriers, and amplitude modulation of the KEK waveform. Figure 5 shows a mountain range plot of cavity voltage used to accumulate five (5) Booster transfers. The BNL cavity was pulsed at the revolution frequency. The KEK cavity had a programmed delay and its amplitude was modulated to minimize emittance growth during coalescence. The first (bottom most) trace was taken about $100 \mathrm{~ms}$ after the first Booster transfer. There was $1.50 \mathrm{~ms}$ between transfers. Figure 6 shows the beam current for the same cycle as Figure 5. The ripples evident during debunching of the first transfer are due to the ripples in

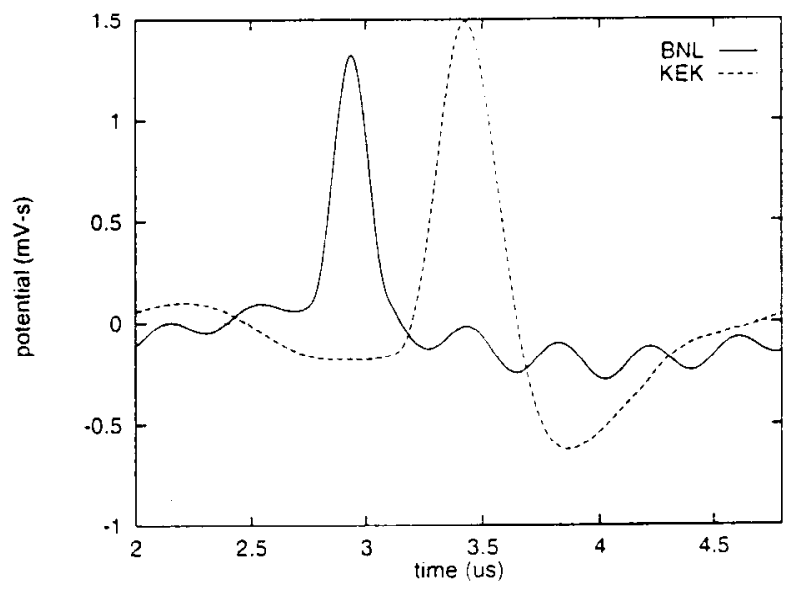

Figure 4: Integral of gap voltage with beam.

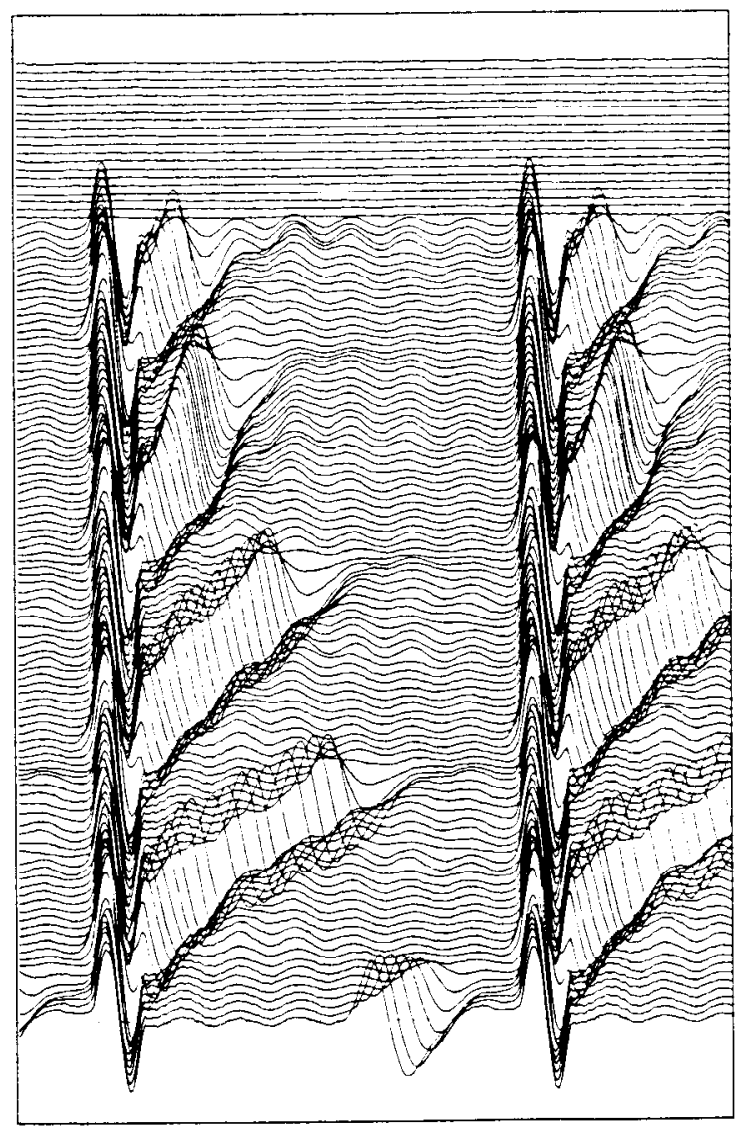

Figure 5: Mountain range plot of gap voltage for five transfers

the BNL waveform, while the tendency for the beam to bunch toward later times is due to the asymmetry in the KEK waveform. After tive transfers were accumulated the beam was rebunched at $h=6$. These bunches had a large longitudinal emittance and large transition losses occurred when the beam was accelerated[ 4 ]. 


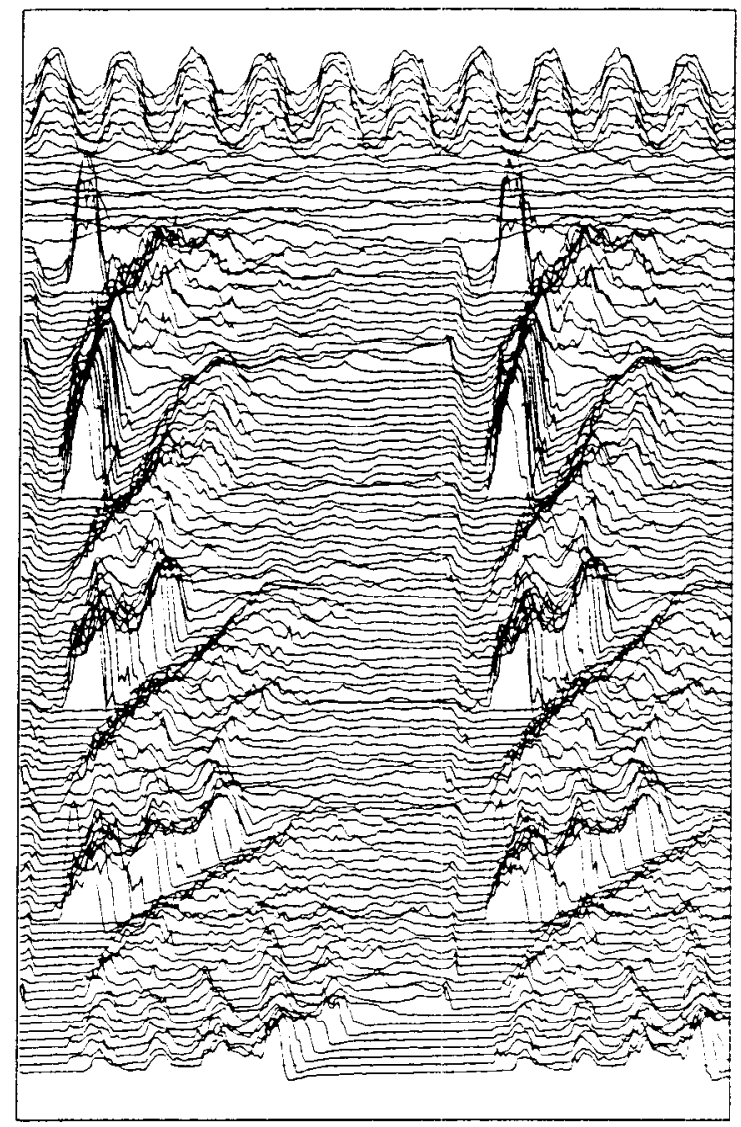

Figure 6: Mountain range plot of beam current for five transfers.

\section{CONCLUSIONS}

The successful operation of a barrier bucket rf systern appears to have several requirements. The barrier voltage pulses must closely approximate single periods of isolated sine waves. Overshoot and ripple will keep the beam from debunching or cause significant emittance growth during the process. The integral of the voltage as in Figures 2 and 4 may be more useful than the voltage itself. A well compensated voltage divider at the cavity is helpful.

Another key feature is the ability to modulate the amplitude of the barrier voltage. Rapidly turning off the waveform results in an emittance growth equal to the product of the width of the barrier and the energy spread of the stored beam.

For emittance conservation the barriers must form a matched bucket for the injected beam. This places constraints on the voltage and frequency that can be more severe than the momentum spread requirements of the debunched beam. Conversely, narrow barriers can place unacceptable constraints on the injection kicker magnet pulse. which was 800 ns in our case.

At moderate to high intensity the effects of beam loading become severe. For a low $Q$ cavity a feedforward system may be adequate but for high $Q$ it is likely that some sort of feedback will be required. Since the barrier voltage is broad band there is no analogy to detuning in a harmonic if system. To cancel the beam induced voltage the power amplifier must be able to deliver the full beam current per accelerating gap. Let $\delta I=I_{b}-I_{f}$ be the difference between the beam current and feedback/feedforward current. In a linear system $\delta I(\omega)=T(\omega) I_{b}(\nu)$ where $T$ is the transfer function for voltage correction. For an RLC resonator with steady state beam loading the mean square error in the integral of the voltage is given by

$$
<\delta L^{2}>=2\left(\frac{R}{Q \omega_{0}}\right)^{2} \sum_{n=1}^{\infty} \frac{\left|T\left(n \omega_{0}\right) I_{b}\left(n \omega_{0}\right)\right|^{2}}{(n / Q)^{2}+\left(n^{2} / h-h\right)^{2}} .
$$

where $h=\omega_{r} / \omega_{0}$ is the generalized harmonic number. The peak drive current needed to create a barrier voltage $V$ is given by [1.2.3] $I_{p}=V(Q+1) / R$ which, for fixed $I_{P}$ and $V^{\circ}$, implies $R / Q=V(1+1 / Q) / I_{p}$.

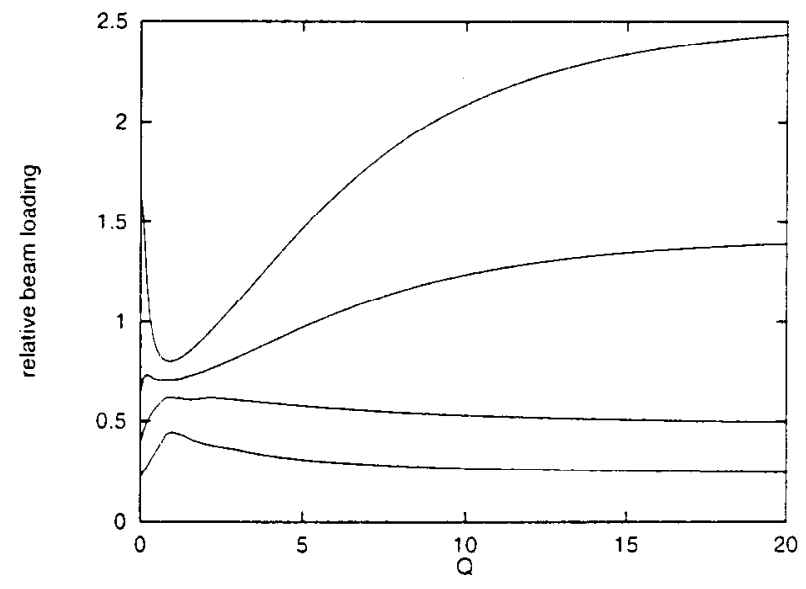

Figure 7: Sum in eq(2) with $T_{n} I_{n}=1$ initially and then removing the largest $0,1,2$ and 4 elements. The harmonic number was $h=7.5$.

For perfect correction $T_{n}=0$ while $T_{n}=1$ for uncorrected lines. To illustrate the interplay of $Q$ with feedback the elements of the sum in equation (2) were calculated with $T_{n} I_{n}=1$, and the value of $R / Q$ was chosen so that the voltage and peak drive current were constant. The sum using all the elements was calculated as were sums with the largest 1.2, or 4 elements set to zero. Figure 7 shows the modified sums versus $Q$ for the four conditions. The optimal value of $Q$ increases with the number of lines corrected.

\section{REFERENCES}

[1] J.E. Griftin. C. Ankenbrandr. J.A. MacLachlan. A. Moretti, IEEE TNS, 30, p3502, 1983

[2] M. Blaskiewicz, AIP conf 377 p283 (1995).

[3] M. Blaskiewicz, J.M. Brennan. EPAC96, p2373 (1996).

[4] M. Fujieda et. al. these proceedings 